\title{
The binding energy and dynamics of charge-transfer states in organic photovoltaics with low driving force for charge separation
}

Cite as: J. Chem. Phys. 150, 104704 (2019); https://doi.org/10.1063/1.5079285

Submitted: 30 October 2018 . Accepted: 19 February 2019 . Published Online: 11 March 2019

Yifan Dong (D), Hyojung Cha (D), Jiangbin Zhang (D), Ernest Pastor (D), Pabitra Shakya Tuladhar, lain McCulloch, James R. Durrant, and Artem A. Bakulin

\section{ARTICLES YOU MAY BE INTERESTED IN}

Theory of coherent two-dimensional vibrational spectroscopy

The Journal of Chemical Physics 150, 100901 (2019); https://doi.org/10.1063/1.5083966

Detailed Balance Limit of Efficiency of p-n Junction Solar Cells

Journal of Applied Physics 32, 510 (1961); https://doi.org/10.1063/1.1736034

Accelerated hole-extraction in carbon-electrode based planar perovskite solar cells by moisture-assisted post-annealing

Applied Physics Letters 114, 103503 (2019); https://doi.org/10.1063/1.5087098

Where in the world is AIP Publishing?

Find out where we are exhibiting next 


\title{
The binding energy and dynamics of charge-transfer states in organic photovoltaics with low driving force for charge separation
}

\author{
Cite as: J. Chem. Phys. 150, 104704 (2019); doi: 10.1063/1.5079285 \\ Submitted: 30 October 2018 - Accepted: 19 February 2019 • \\ Published Online: 11 March 2019
}

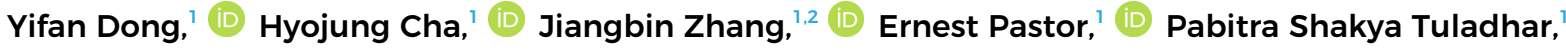
lain McCulloch, ${ }^{1,3}$ James R. Durrant, ${ }^{1,4, a)}$ and Artem A. Bakulin ${ }^{1, a)}$
\end{abstract}

\begin{abstract}
AFFILIATIONS
${ }^{1}$ Department of Chemistry and Centre for Plastic Electronics, Imperial College London, London SW7 2AZ, United Kingdom

${ }^{2}$ Cavendish Laboratory, University of Cambridge, JJ Thomson Avenue, Cambridge CB3 OHE, United Kingdom

${ }^{3}$ Physical Sciences and Engineering Division, KAUST Solar Centre (KSC), King Abdullah University of Science and Technology (KAUST), Thuwal 23955-6900, Saudi Arabia

${ }^{4}$ SPECIFIC IKC, College of Engineering, Swansea University, Swansea SA12 7AX, United Kingdom
\end{abstract}

Note: This article is part of the Special Topic "Nonlinear Spectroscopy and Interfacial Structure and Dynamics" in J. Chem. Phys. a) Authors to whom correspondence should be addressed: j.durrant@imperial.ac.uk and a.bakulin@imperial.ac.uk

\begin{abstract}
Recent progress in organic photovoltaics (OPVs) has been enabled by optimization of the energetic driving force for charge separation, and thus maximization of open-circuit voltage, using non-fullerene acceptor (NFA) materials. In spite of this, the carrier dynamics and relative energies of the key states controlling the photophysics of these systems are still under debate. Herein, we report an in-depth ultrafast spectroscopic study of a representative OPV system based on a polymer donor PffBT4T-2OD and a small-molecule NFA EH-IDTBR. Global analysis of the transient absorption data reveals efficient energy transfer between donor and acceptor molecules. The extracted kinetics suggest that slow ( 15 ps) generation of charge carriers is followed by significant geminate recombination. This contrasts with the "reference" PffBT4T-2OD:PC ${ }_{71}$ BM system where bimolecular recombination dominates. Using temperature-dependent pump-push-photocurrent spectroscopy, we estimate the activation energy for the dissociation of bound charge-transfer states in PffBT4T-2OD:EH-IDTBR to be 100 $\pm 6 \mathrm{meV}$. We also observe an additional activation energy of $14 \pm 7 \mathrm{meV}$, which we assign to the de-trapping of mobile carriers. This work provides a comprehensive picture of photophysics in a system representing new generation of OPV blends with a small driving force for charge separation.
\end{abstract}

Published under license by AIP Publishing. https://doi.org/10.1063/1.5079285

\section{INTRODUCTION}

Most organic photovoltaic (OPV) devices employ the bulkheterojunction architecture with an interpenetrating network of electron donor (D) and electron acceptor (A) organic molecules as the absorber materials. ${ }^{7}$ While the role of $\mathrm{D}$ materials has been traditionally played by a wide variety of conjugated polymers, the role of acceptors was usually taken by fullerene and its derivatives, such as $[6,6]$-phenyl-C71-butyric acid methyl ester $\left(\mathrm{PC}_{71} \mathrm{BM}\right)$. However, over the past few years, a new class of small molecules known as non-fullerene acceptors (NFAs) have been developed to replace fullerenes. ${ }^{2-7}$ When mixed with common polymer donors including P3HT, PTB7-Th, and PffBT4T-2OD, NFA-based blends show enhanced open-circuit voltage $\left(V_{O C}\right)$ and increased stability compared to their fullerene counterparts. ${ }^{6,8-14}$ The most efficient singlejunction OPV to date has reached a power conversion efficiency of $14 \%$ using the NFA ITIC-4F. ${ }^{15}$ Despite this rapid rise in efficiency, a fundamental understanding of the charge generation mechanism is still lagging behind.

Bound electron-hole pairs, excitons, are generated in D and A materials upon photoexcitation. Excitons dissociate at the D/A interface and form interfacial charge-transfer states (CTSs). 
This process can be driven by the energetic difference between the lowest unoccupied molecular orbitals (LUMOs) or the highest occupied molecular orbitals (HOMOs) of D and A. It has been widely reported that free charges can be generated from these intermediate CTSs, leading to photocurrent generation in devices. However, the detailed mechanism of charge separation from CTSs remains controversial. ${ }^{16-19}$ The energy of the CTS, $\mathrm{E}_{\mathrm{CT}}$, is related to the energetic difference between the HOMO of D and the LUMO of A, which limits the attainable $V_{O C} .^{20}$ The remarkably high $V_{O C}$ in NFA-based blends has been achieved by increasing $\mathrm{E}_{\mathrm{CT}}$ via matching the LUMO or HOMO level of the acceptor closer to that of D. ${ }^{21-23}$ This implies that a smaller driving force for charge separation is a common feature among the state-of-the-art NFA-based OPVs. ${ }^{24,25}$ Understanding how the smaller driving force affects the dynamics of CTSs is hence crucial to improve the charge generation in NFA-based OPVs.

One of the NFA-based benchmark systems with a low driving force for charge separation is PffBT4T-2OD:EH-IDTBR. PffBT4T$2 \mathrm{OD}$ is a polymer donor which was first reported by Yan and coworkers. ${ }^{26}$ It exhibits strong aggregation ability and tends to form highly crystalline domains. ${ }^{26}$ The NFA EH-IDTBR was reported by the McCulloch group in 2016. ${ }^{10}$ It is based on an indacenodithiophene core with ethyl hexyl side chains. The planar conjugation together with the highly electron-rich core raises the LUMO, thereby increasing $\mathrm{E}_{\mathrm{CT}}$ relative to fullerene-based blends. EH-IDTBR also exhibits a high molar extinction coefficient $\left(\sim 10^{5} \mathrm{M}^{-1} \mathrm{~cm}^{-1}\right)$ in solutions and a higher absorption coefficient in thin films than some polymer donors. ${ }^{10}$ This opens up the possibility for hole transfer to be as equally important as electron transfer for charge generation in devices.

In our previous work on PffBT4T-2OD:EH-IDTBR and PffBT4T-2OD:PC ${ }_{71} \mathrm{BM}$, we reported relatively long exciton quenching times $(30 \pm 2 \mathrm{ps})$ in both systems that are still much shorter than the exciton lifetime in the neat PffBT4T-2OD (300 ps). ${ }^{9}$ This agrees with the photoluminescence (PL) quenching yield (77\% and $80 \%$ for EH-IDTBR and $\mathrm{PC}_{71} \mathrm{BM}$ systems, respectively). Although similar exciton quenching kinetics and yields were observed, the yield of free carriers in the former system is lower, leading to a drop in short-circuit current $\left(J_{S C}\right)$. Cha et al. attributed this to the smaller driving force for charge separation and a greater degree of geminate recombination in PffBT4T-2OD:EH-IDTBR. ${ }^{9,27}$ However, in-depth analysis of the transient absorption (TA) data has not been performed. The exciton and charge dynamics as well as the dynamics of CTS have not been clearly identified. ${ }^{9}$ The enhanced stability and $V_{O C}$ together with the congruent absorption between PffBT4T-2OD and EH-IDTBR make their blend an interesting system for in-depth spectroscopic studies. Previous studies have indicated the promising potential of OPV blends with overlapping absorption features. For example, Baran et al. have reported that blending PTB7-Th with EH-IDTBR leads to stable and efficient OPV with minimal recombination losses. ${ }^{8}$ Also Kirchartz et al. have recently pointed out overlapping absorption is preferable and beneficial for OPV performance if the active layer thickness is relatively thin, which is commonly observed among NFA-based OPVs. ${ }^{28}$

This study goes beyond the previous work and focuses on investigating the carrier dynamics in PffBT4T-2OD:EH-IDTBR with different types of ultrafast spectroscopy tools and advanced global analysis of the TA data. The use of global analysis identifies the spectral footprints for PffBT4T-2OD excitons, EH-IDTBR excitons, and charges. It reveals the ultrafast energy transfer between PffBT4T2OD and EH-IDTBR excitons under photoexcitation. However, the subsequent exciton dissociation into CTSs appears to be slow ( $\sim 15$ ps). Rather than separating into free charges, a large number of CTSs remain bound and undergo geminate recombination. This differs from the "reference" PffBT4T-2OD:PC ${ }_{71} \mathrm{BM}$ system where charge separation is also efficient but is followed by bimolecular recombination. To understand the nature of CTSs in PffBT4T2OD:EH-IDTBR, we investigate how the temperature affects charge generation using pump-push-photocurrent (PPPC) spectroscopy. PPPC measurements were employed to address the dynamics of interfacial bound states in OPVs. The early time $(<100$ ps) PPPC kinetics resemble the CTS dynamics extracted from the TA data. This implies that the same photophysical process governs the formation of CTSs. We further used the Arrhenius relation to fit the early time PPPC traces across different temperatures to determine the activation energy $\left(\mathrm{E}_{\mathrm{a}}\right)$ for CTS formation to be on the order of $100 \pm 6 \mathrm{meV}$, which is the first reported $E_{a}$ value for NFA blends. In addition, by fitting the long-time PPPC kinetics, the activation energy of charge de-trapping was determined to be 14 $\pm 7 \mathrm{meV}$.

\section{MATERIALS}

Figure 1(a) displays the absorption spectra of PffBT4T-2OD, EH-IDTBR, $\mathrm{PC}_{71} \mathrm{BM}$, and their blends. PffBT4T-2OD shows a
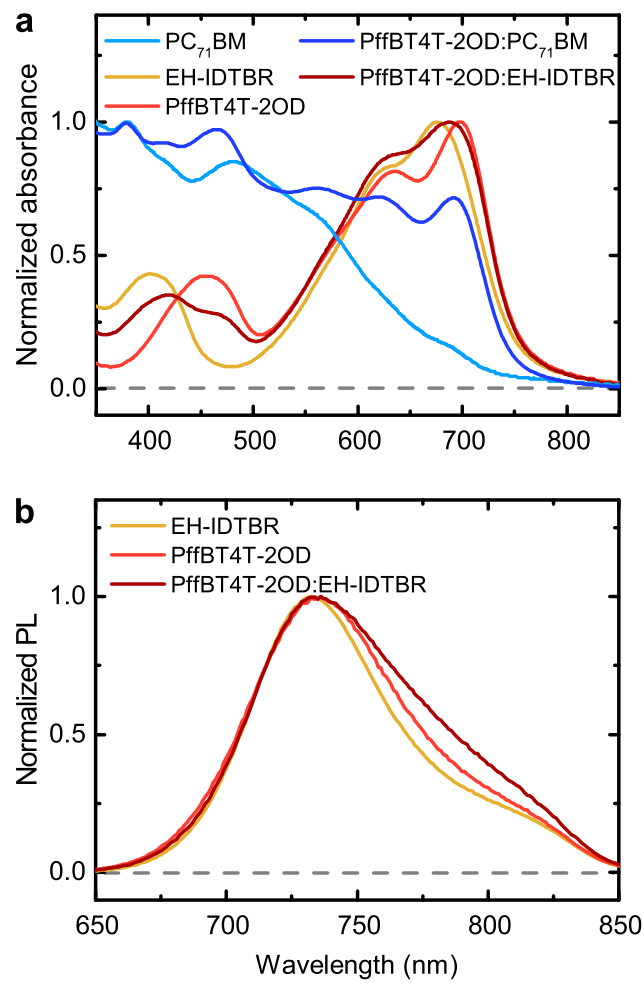

FIG. 1. (a) Normalized steady-state absorption spectra of the materials under study. (b) Normalized steady-state PL spectra for PffBT4T-2OD, EH-IDTBR, and PffBT4T-2OD:EH-IDTBR blend. All samples were excited at $600 \mathrm{~nm}$. 
pronounced absorbance peak at $700 \mathrm{~nm}$, which has been previously assigned to the strong aggregation in the film. ${ }^{26} \mathrm{EH}$-IDTBR exhibits an absorbance peak at a similar wavelength $(675 \mathrm{~nm})$. The absorption spectra of these two neat materials largely overlap between 500 and $800 \mathrm{~nm}$. Figure 1(b) shows the normalized steady-state PL spectra for PffBT4T-2OD, EH-IDTBR, and their blend. PffBT4T$2 \mathrm{OD}$ and $\mathrm{EH}-\mathrm{IDTBR}$ emit at 732 and $736 \mathrm{~nm}$, respectively, in agreement with their previously reported bandgap values (1.69 eV and $1.68 \mathrm{eV})$. After mixing PffBT4T-2OD with EH-IDTBR, the PL emission maximum peak is broadened and shows a "flat" peak between 732 and $736 \mathrm{~nm}$. This indicates the blend emission comes from the excitons in both materials. The blend PL resembles both neat materials in the 650-736 $\mathrm{nm}$ region, while the lower energy tail $(736-850 \mathrm{~nm})$ is expanded and is probably due to the increase in disorder upon mixing. Figure S3 compares the steady-state PL with the time-resolved PL taken from time-correlated single photon counting (TCSPC) for neat PffBT4T-2OD, neat EH-IDTBR, and their blend. For each material and their blend, both the steady-state and the timeresolved PL spectra resemble each other. Additionally, the emission maxima stay unchanged at different time delays (0-100 ns) for all the samples. While the emission in the neat materials is dominated by excitons, the emission in the blend could be from both excitons and CTSs. The same PL emission peak in the blend hence suggests two possibilities: (1) the excitons and CTS emit at the same energy, or (2) the radiative emission from CTSs is relatively weak to be observed. Previous studies have indicated that the PffBT4T-2OD:EH-IDTBR system has small LUMO and HOMO energy differences of 0.21 and $0.24 \mathrm{eV}$, respectively, between $\mathrm{D}$ and $\mathrm{A},{ }^{9}$ leading to a lower CTS energy than the excitons. However, the broadening of the lower energy tail $(750 \mathrm{~nm}-850 \mathrm{~nm})$ may represent the contribution from the CTSs. The fact that D and A have a low exciton energy difference $(0.01 \mathrm{eV})$ makes it challenging to distinguish which material dominates the charge generation in the blend. Also, a significant number of excitons have been quenched by 100 ps which is below the time resolution of the TCSPC instrument. Therefore, we now turn to the TA data to investigate exciton separation and charge recombination kinetics over the ps to ns time scale.

\section{RESULTS AND DISCUSSION}

\section{A. Transient absorption spectroscopy}

Figure 2 compares the time-resolved near-infrared (900 $-1400 \mathrm{~nm}$ ) TA spectra of the two OPV blends. For the PffBT4T2OD:PC ${ }_{71}$ BM blend film in Fig. 2(a), the initial PIA peak at $1100 \mathrm{~nm}$ is assigned to the PffBT4T-2OD exciton. This is confirmed by comparison with the neat PffBT4T-2OD TA data (Fig. S4). In neat PffBT4T-2OD, the exciton PIA peak stays at $1100 \mathrm{~nm}$ with a minimal spectral shift over time, indicating that only PffBT4T-2OD excitons are present. In the PffBT4T-2OD:PC ${ }_{71} \mathrm{BM}$ blend shown in Fig. 2(a), the PIA peak remains at $1100 \mathrm{~nm}$, but the lower energy shoulder (1100-1400 nm) becomes narrower within the first $100 \mathrm{ps}$. This distortion in the blend spectra indicates the appearance of another species while the PffBT4T-2OD exciton decays. Because only PffBT4T-2OD was excited, charges formed through photoinduced electron transfer from PffBT4T-2OD to $\mathrm{PC}_{71} \mathrm{BM}$ are the most likely species. After 100 ps, there is no further change in the spectra. Therefore, we attribute the PIA after 100 ps solely to the charges since all the excitons have decayed.

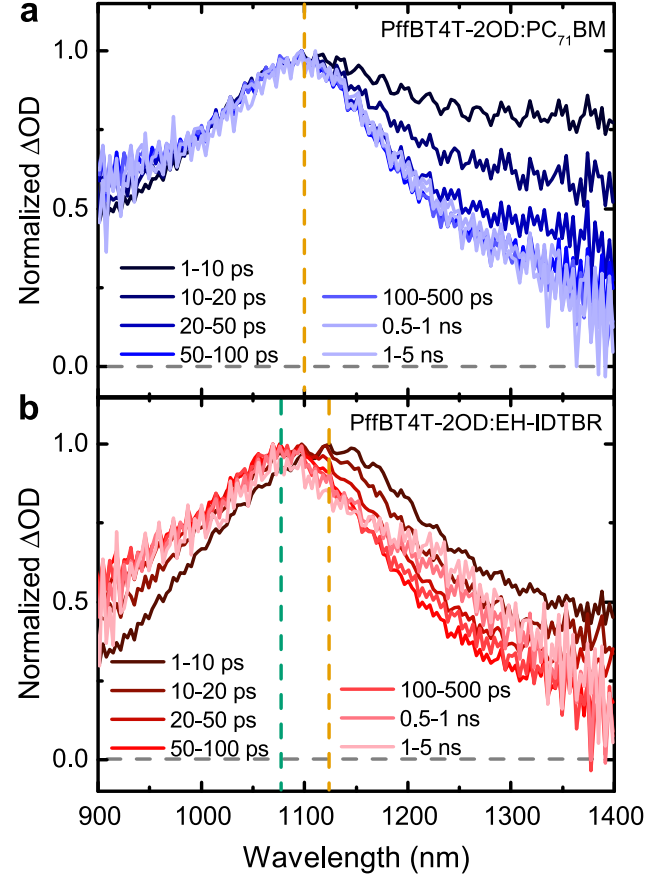

FIG. 2. (a) Normalized TA spectra for the PffBT4T-2OD:PC ${ }_{71} B M$ blend film at different pump-probe delay times. The orange dashed line indicates the same PIA peak at $1100 \mathrm{~nm}$ throughout the measurement. (b) Normalized TA spectra for the PffBT4T-2OD:EH-IDTBR blend film at different pump-probe delay times. The orange and green dashed lines represent the initial PIA peak at $1125 \mathrm{~nm}$ and the evolved PIA peak at $1080 \mathrm{~nm}$. The pump wavelength was $700 \mathrm{~nm}$, and the fluence was $10 \mu \mathrm{J} \mathrm{cm}^{-2}$ for both samples.

Figure 2(b) shows the TA data for the PffBT4T-2OD:EHIDTBR blend film. The initial PIA peak stays at $1125 \mathrm{~nm}$, whereas the PIA peaks for neat PffBT4T-2OD and neat EH-IDTBR are at $1100 \mathrm{~nm}$ and $1150 \mathrm{~nm}$, respectively. Additionally, the spectra shape at 1 ps differs from either one of the neat materials' spectra (Fig. S6). Therefore, we assign the PIA peak at $1125 \mathrm{~nm}$ in the blend to the combination of exciton absorption from both materials due to the co-excitation under the laser pump. This PIA peak gradually shifts to $1080 \mathrm{~nm}$ over $50 \mathrm{ps}$. This is due to the photoinduced electron and hole transfer between PffBT4T-2OD and EH-IDTBR excitons. The co-existence of D and A excitons in NFA OPV blends has also been reported elsewhere and is becoming a common feature among NFA-based OPV systems. ${ }^{24,29}$ For example, Laquai and co-workers observed the co-existence of cations and anions in TA formed through electron and hole transfer in PTB7-Th:CDTBM. ${ }^{30}$ However, few studies indicate the individual dynamics of $D$ and $A$ exciton decay. To further understand the charge carrier dynamics of each species, we use global analysis to deconvolute the kinetics of individual excited state species from the TA data.

\section{B. Global analysis of TA}

Figures 3(a) and 3(b) show the deconvoluted spectra and the corresponding kinetics for the PffBT4T-2OD:PC ${ }_{71} \mathrm{BM}$ blend film using global analysis. The results clearly indicate the presence of 

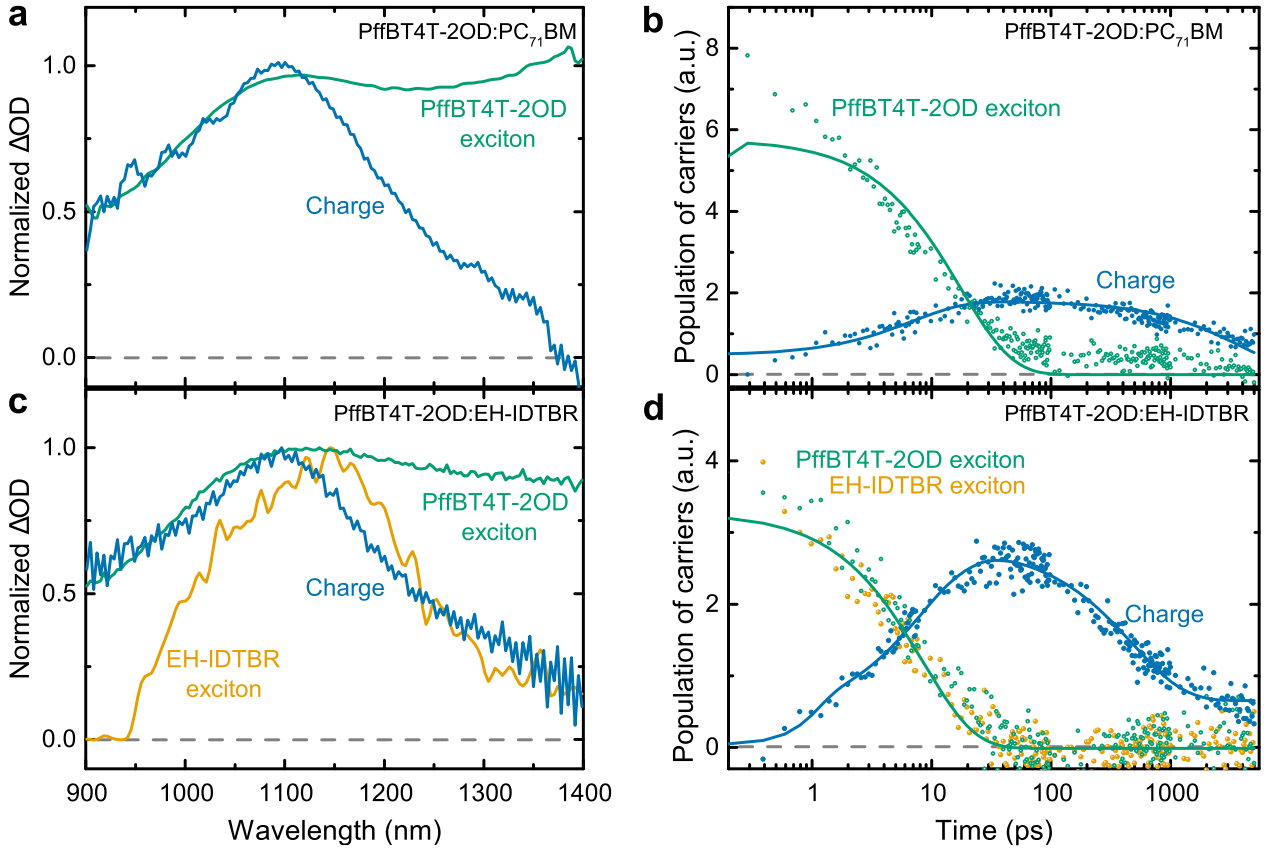

FIG. 3. Deconvoluted spectra [(a) and (c)] and kinetics [(b) and (d)] for PffBT4T2OD: PC ${ }_{71} B M$ and PffBT4T-2OD: EHIDTBR blends. Samples were excited at $700 \mathrm{~nm}$ with a fluence of $10 \mu \mathrm{J} / \mathrm{cm}^{2}$ two excited state species when PffBT4T-2OD was selectively excited by pumping at $700 \mathrm{~nm}$. By comparing the spectra with the neat PffBT4T-2OD spectra (Fig. S4), we assign the broad spectra feature centred at $1100 \mathrm{~nm}$ to PffBT4T-2OD excitons. The kinetics in Fig. 3(b) show one component with a fast decay rate and another component being gradually formed which exhibits a longer lifetime. The fast-decaying component is PffBT4T-2OD excitons, and the faster decay kinetics relative to that in neat PffBT4T-2OD TA represents the quenching due to the electron transfer to $\mathrm{PC}_{71} \mathrm{BM}$. The other component is hence assigned to the charges that are generated as a result of charge transfer. Fitting the kinetics in Fig. 3(b) yields a quenching time of 15 ps for PffBT4T-2OD excitons. A notable feature here is the slow formation of charges which was also observed before in TA for the same blend. ${ }^{31}$ This is in contrast with other well intermixed fullerene-based OPV systems where charge generation is much faster ( $200 \mathrm{fs}))^{32-34}$ The slow generation here may be associated with the relatively large domain size of PffBT4T-2OD aggregates in the PffBT4T-2OD:PC 71 BM blend as shown in the TEM (Fig. S7). ${ }^{9}$

Similarly, Figs. 3(c) and 3(d) show the deconvoluted spectra and the corresponding kinetics for each species in the PffBT4T2OD:EH-IDTBR blend. The deconvoluted spectra in Fig. 3(c) indicate three distinct PIA spectra centred at 1080, 1100, and $1150 \mathrm{~nm}$ individually. As discussed earlier, both PffBT4T-2OD and EH-IDTBR excitons are present under illumination. Comparing with the neat material spectra (Figs. S4 and S5), we therefore assign the broad PIA feature centring at $1100 \mathrm{~nm}$ to PffBT4T-2OD excitons and the narrower PIA at $1150 \mathrm{~nm}$ to EH-IDTBR excitons. As shown in the TA data [Fig. 2(b)], the PIA peak of the long-lived charges shifts to $1080 \mathrm{~nm}$. We therefore assign the third component with an absorption peak at $1080 \mathrm{~nm}$ to the feature of charges. Turning to the kinetics in Fig. 3(d), both PffBT4T-2OD and EH-IDTBR excitons decay with the same quenching time of $9 \mathrm{ps}$, comparable to previous studies. ${ }^{9}$ The same decay time of both excitons provides evidence that the PffBT4T-2OD exciton and EH-IDTBR exciton are going through ultrafast energy transfer due to their similar exciton energies ( 1.68 and $1.69 \mathrm{eV}$, respectively). While the excitons decay, charges are generated with a similar lifetime of $10-15$ ps and subsequently decay with a lifetime of $1.2 \mathrm{~ns}$. Although the TEM shows an intermixed morphology in the PffBT4T2OD:EH-IDTBR blend (see TEM in Fig. S7), the quenching time of excitons and the generation time of charges still happen on a shorter time scale compared to other finely mixed fullerene blends, in which the excitons typically dissociate on an ultrafast ( $200 \mathrm{fs}$ ) time scale. $^{35-37}$

We note that the yield of charges at 6 ns is relatively low in the PffBT4T-2OD:EH-IDTBR blend (only 25\% of charges escape the recombination pathway and contribute to the long-lived charge generation). One possible reason is the acceleration of the exciton and charge dynamics due to the exciton-exciton or exciton-charge annihilation at slightly high pump fluences. To exclude this contribution and to distinguish which recombination pathway dominates for each blend, we also performed the same TA measurements and global analysis under various pump fluences.

Figure 4(a) displays the charge dynamics at various pump fluences extracted from global analysis for PffBT4T-2OD:PC ${ }_{71} B M$. As the pump fluence decreases, the amplitude representing the yield of charges drops proportionally. While the generation kinetics are mostly independent on the pump fluence, the recombination kinetics are strongly fluence-dependent. At a lower pump fluence, we observe a much slower decay with more charges remaining across our measurement time scale. The charge lifetime gradually increases from 0.8 to $15 \mathrm{~ns}$ as the fluence decreases from 20 to $2.5 \mu \mathrm{J} / \mathrm{cm}^{2}$. From the fluence-dependent dynamics, it indicates negligible 


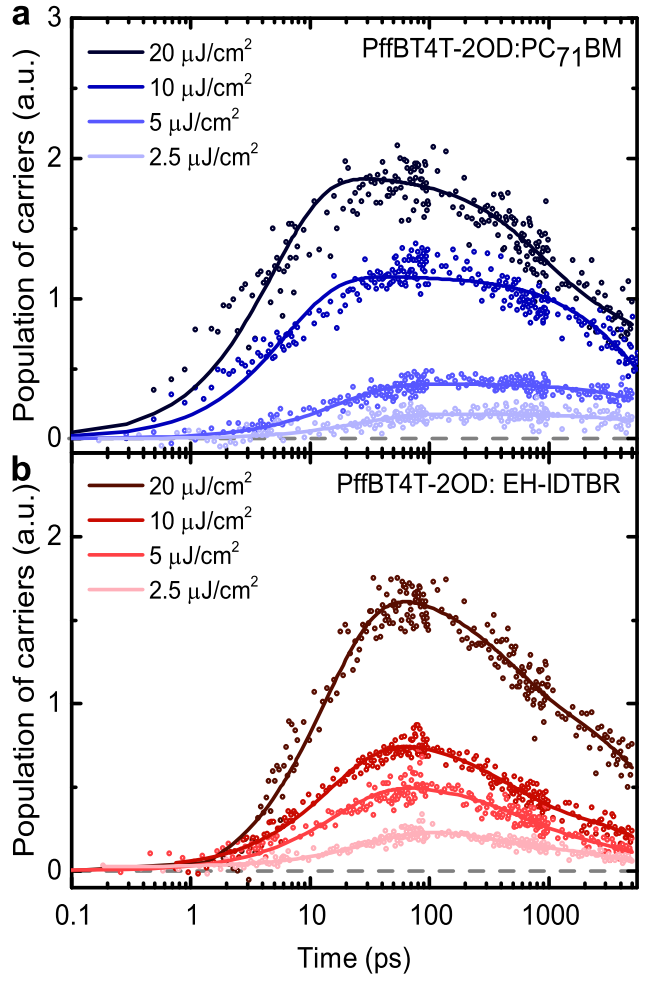

FIG. 4. Deconvoluted kinetics of charges for (a) PffBT4T-2OD:PC ${ }_{71} B M$ blend and (b) PffBT4T-2OD:EH-IDTBR at different pump fluences from global analysis. Solid lines are multiexponential fittings as guide to the eyes.

geminate losses and the main loss is the bimolecular recombination of charges as observed by Cha et al. ${ }^{9}$ Figure 4(b) displays the charge kinetics for PffBT4T-2OD:EH-IDTBR extracted from global analysis for TA carried out at various fluences. When compared to the $\mathrm{PC}_{71} \mathrm{BM}$ blend, here the charge generation time is slower $(\sim 15 \mathrm{ps})$ at the lowest fluence of $2.5 \mu \mathrm{J} / \mathrm{cm}^{2}$ ). The recombination kinetics show fluence-independent behavior, which indicates that the geminate recombination pathway dominates. A significant amount of charges are already recombined by $5 \mathrm{~ns}$ even at a lower fluence similar to 1 Sun condition $\left(2.5 \mu \mathrm{J} / \mathrm{cm}^{2}\right)$. Comparing the kinetics of charges in these two blends at the same fluence, we always observe a higher recombination loss and a faster decay kinetics in the PffBT4T-2OD:EH-IDTBR blend.

\section{Pump-push-photocurrent spectroscopy}

Pump-push-photocurrent (PPPC) measurements have previously been employed to probe the dynamics of bound CTSs at molecular interfaces. PPPC is hence employed here to address the dissociation dynamics of CTSs by probing the photocurrent change in PffBT4T-2OD:EH-IDTBR devices. In OPVs, charge transfer of excitons at the D/A interface generates CTSs which then go through relaxation to localized states. The successful escape of the electron and hole from CTSs generates free carriers, and this dissociation efficiency is directly related to the photocurrent in devices. ${ }^{38}$ In PPPC measurements, the yield of free carriers and the charge extraction efficiency $\left(\eta_{\text {ext }}\right)$ determine the reference current $(J)$ which is measured when the cell is illuminated by the $650 \mathrm{~nm}$ pump beam only. The later arrival of the IR push pulse $(2000 \mathrm{~nm})$ excites the localized bound CTSs into higher energy states and assists the dissociation of CTSs into free carriers with a certain probability $\left(P_{d i s}\right)$. For technical reasons, $2000 \mathrm{~nm}$ push was used. However, the dynamics at both $1300 \mathrm{~nm}$ and $2000 \mathrm{~nm}$ push appeared to be similar as shown in our previous work. ${ }^{31}$ Therefore, we assign the signal to the optical activation of localized immobile charged states that can be CTS or separated carriers in the shallow traps. As a result of this push beam, extra photocurrent $(\Delta J)$ is generated. The additional photocurrent, $\Delta J$, is linked with the product of the number of localised CTSs $\left(N_{C T}\right)$, the dissociation probability $\left(P_{d i s}\right)$, and the charge extraction efficiency $\left(\eta_{\text {ext }}\right) .{ }^{39}$ Varying the time delays between the pump and push pulses up to 2 ns, we can monitor how the population of bound CTSs changes as a function of time.

Figure 5(a) represents the early time PPPC kinetics for the PffBT4T-2OD:EH-IDTBR device. We narrow our analysis down to the first 100 ps time window where the kinetics show a slow rise with a time constant of 15 ps. We attribute this signal to the formation of localized CTSs. The slow generation of CTSs has been reported recently for other OPV systems. ${ }^{21,37,40}$ As mentioned before, for the $\mathrm{PC}_{71} \mathrm{BM}$ case, the slow diffusion of excitons across the relatively large PffBT4T-2OD domains could be one of the reasons for this slow generation of CTSs. ${ }^{9,41}$ Another possible reason could be the high activation barrier to form CTSs, which will be examined later. In any case, the similarity between the TA kinetics and the PPPC kinetics indicates that CTS generation is governed by the same mechanism in films and devices.

Figure 5(b) represents the PPPC kinetics at different temperatures for the PffBT4T-2OD:EH-IDTBR device. Both the amplitude $(\Delta J / J)$ and kinetics depend on $T$. To understand the $T$-effect, we examine kinetics in two separate time regimes: before 100 ps and after 500 ps. The signal before 100 ps is likely to be associated with freshly generated bound CTSs. As expected, the peak amplitude increases as $T$ decreases, reflecting an increase in the population of CTSs. At lower temperatures, fewer CTSs can overcome the electrostatic barrier and directly dissociate into free carriers. Therefore, the push pulse has a greater impact at low temperatures since there are more bound CTSs that can be dissociated, hence increasing the amplitude of PPPC signal. For a more quantitative analysis of the $T$-dependence, we apply an Arrhenius plot approach as shown in Fig. 5(c). Based on our previous work, ${ }^{42}$ we extracted the amplitude at early time from Fig. 5(b) and plotted it against the reciprocal $T$. The dependence can be described by two activation energies. At temperatures above $200 \mathrm{~K}$, the dominant process responsible for the charge trapping has an $\mathrm{E}_{\mathrm{a}}$ of $100 \mathrm{meV}$, which is attributed to the binding energy of CTSs. Below $200 \mathrm{~K}$, the behavior changes, and carrier extraction is limited by a different process with an $E_{a}$ of $14 \mathrm{meV}$ limiting carrier extraction, which is discussed further below.

After a few hundred ps, the signal flattens out (and even exhibits minor growth), which was previously attributed to charge trapping. ${ }^{43}$ Indeed, the $T$-dependence of the PPPC signal measured at 600 ps reveals a lower activation energy of $14 \mathrm{meV}$ which is typical for the trapping of free carriers during charge transport. Although the push pulse has a minimal effect on mobile carriers near the band edge, ${ }^{42}$ it is able to elevate free carriers from low energy states to 

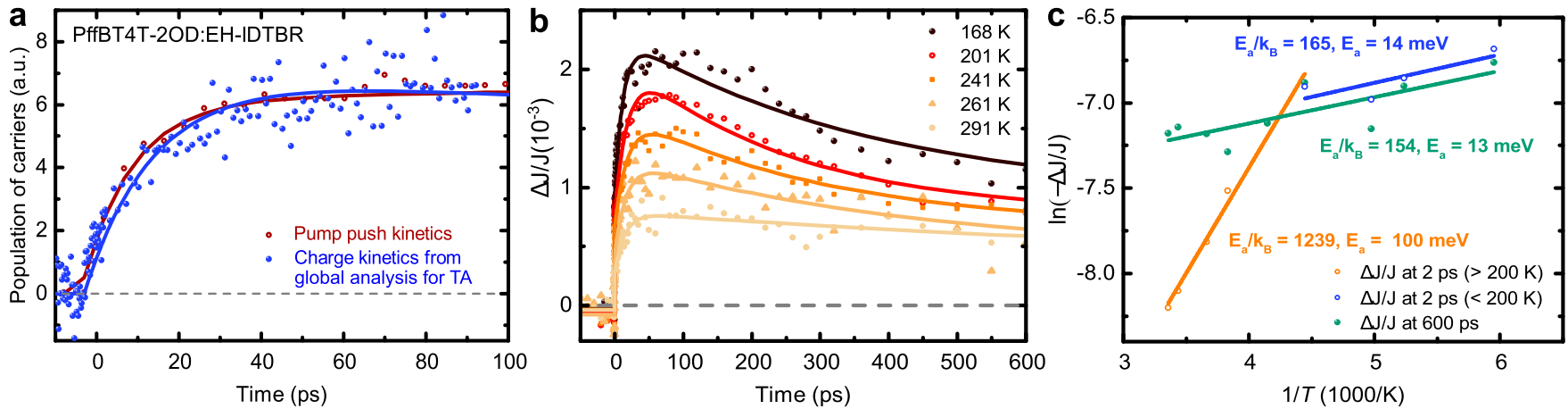

FIG. 5. (a) Comparing the early time pump-push-photocurrent kinetics (red) and the deconvoluted charge generation kinetics (blue) extracted from the TA data for the PffBT4T2OD:EH-IDTBR device. Samples were excited at $650 \mathrm{~nm}$ and measured at room temperature. (b) T-dependent pump-push-photocurrent kinetics for PffBT4T-2OD:EH-IDTBR between $168 \mathrm{~K}$ and $291 \mathrm{~K}$. (c) Arrhenius plot of the In value of the $\Delta \mathrm{J} / \mathrm{J}$ amplitude at $2 \mathrm{ps}$ [extracted from Fig. 5(b)] as a function of the reciprocal $T$ represented by the circle symbols. Solid lines indicate the linear fitting based on the equation $\ln \left(-\frac{\Delta I}{J}\right)=-\frac{E_{a}}{k_{B} T}$, where $k_{B}$ is the Boltzmann constant. Linear fittings in the high temperature regime (the orange solid line) and the low temperature regime (the blue solid line) yield an activation energy of 100 and $14 \mathrm{meV}$, respectively.

mobilize them again and thus generate additional photocurrent. ${ }^{43}$ We therefore attribute this late-time signal to the de-trapping of separated charges. We note that this $\mathrm{E}_{\mathrm{a}}$ is the same as the $14 \mathrm{meV}$ observed for early time data at temperatures below $200 \mathrm{~K}$. We therefore conclude that at low temperatures, carrier trapping can be as important for charge separation as the CTS binding energy.

Evaluation of the activation energy has been a long-standing question in application to polymer-polymer and polymer-fullerene OPVs, and various values have been reported based on different techniques. For example, Gélinas et al. have reported a binding energy of $250 \mathrm{meV}$ for CTSs in the PFB:F8BT system using PL and TA over the ns time window. ${ }^{44}$ Gao et al. have also interpreted the activation energy for charge separation to be between 9 and $25 \mathrm{meV}$ depending on the morphology of the P3HT: $\mathrm{PC}_{60} \mathrm{BM}$ with External quantum efficiency (EQE). ${ }^{16}$ More recently, Neher and co-workers have reported the activation energy for charge formation from thermalized CTSs to be around $25 \mathrm{meV}$ using time-delayed collection field (TDCF). ${ }^{19}$ The activation energy values vary depending on the technique and the time scale selected. In our work, we probe the CTS behavior at relatively early times, exclusively involving charge generation from bound states. This differs from values extracted from other techniques that usually address a steady-state material/device performance that is controlled by a combination of electronic processes. We realize the reported $100 \mathrm{meV}$ dissociation energy of CTSs is similar to other fullerene systems, which indicates the smaller driving force has little effect of generation of CTSs. In some fullerenebased blends, charge generation also shows independence on the energetic offsets. ${ }^{45}$ Herein, we present the first report of binding energy for CTSs in NFA-based OPVs with a small driving force for charge separation.
Exciton

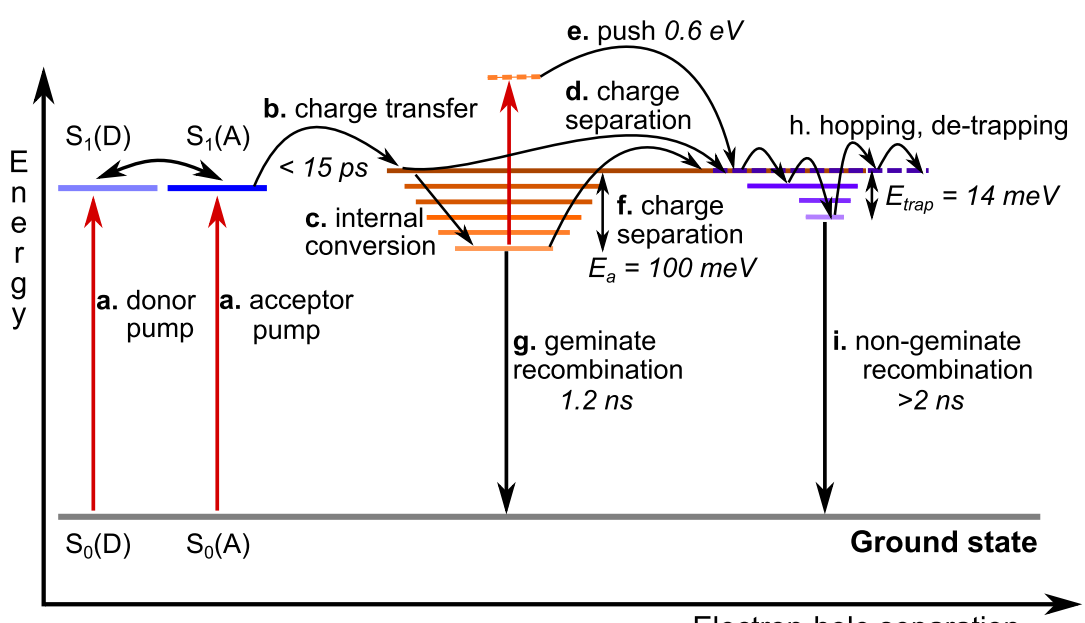

Electron-hole separation
FIG. 6. Proposed model for charge carrier dynamics in OPV blends with a small driving force for charge separation and overlapping absorption profiles. In the PffBT4T-2OD:EH-IDTBR blend: (a) Both $D$ and $A$ excitons exist upon photoexcitation and undergo energy transfer with each other. (b) Dissociation of excitons at the interface to form CTSs. CTSs can either (c) relax into localized states or (d) separate into free carriers. [(e) and (f)] The push pulse $(0.6 \mathrm{eV})$ excites localized CTSs to higher energetic states and dissociates them into free carriers. This dissociation has an activation energy of $100 \mathrm{meV}$. (g) Geminate recombination of CTSs governs the main recombination pathway in this blend. (h) Carriers hop and de-trap with a trap depth of $14 \mathrm{meV}$. (i) Carriers will go through bimolecular recombination if not being extracted out of the electrodes. 
Bringing together the PL, TA, and PPPC results, we propose Fig. 6 as a model for charge generation in PffBT4T-2OD:EH-IDTBR. Upon illumination, (a) excitons are formed in both PffBT4T-2OD and EH-IDTBR. Due to the similar singlet exciton energies, energy transfer occurs between PffBT4T-2OD and EH-IDTBR. (b) Excitons dissociate at the D/A interface within 15 ps to form bound CTSs. (c) A significant fraction of the initially formed CTSs undergo rapid internal conversion to lower localized states. (d) Another portion of the initially formed CTSs directly separates into free carriers. (e) When the push pulse arrives, localized CTSs can be excited to higher energy states to dissociate into free carriers. (f) The subsequent dissociation of CTSs into free carriers happens with different efficiencies depending on $T$. The activation barrier has been found to be $100 \mathrm{meV}$ from $T$-dependent PPPC measurements. (g) A portion of CTSs remains bound and recombines over 1.2 ns. (h) Free carriers transport including trapping, de-trapping, and hoping between different states. The trap depth has been estimated to be $14 \mathrm{meV}$. (i) Free carriers can then undergo bimolecular recombination or extraction to the electrodes.

\section{CONCLUSION}

In conclusion, we employed two spectroscopy tools, TA and PPPC, to elucidate the charge carrier dynamics in a NFA OPV blend PffBT4T-2OD:EH-IDTBR. The results indicate rapid energy transfer between PffBT4T-2OD excitons and EH-IDTBR excitons following photoexcitation. The dissociation of excitons at the interface generates CTSs slowly with a time constant of $15 \mathrm{ps}$. We have also clarified that the same mechanism governs the charge generation in both blend films and devices. The main loss mechanism in the PffBT4T-2OD:EH-IDTBR blend is geminate recombination of charges occurring on the time scale of $1 \mathrm{~ns}$. T-dependent PPPC suggests a relatively low CT exciton binding energy of $100 \pm 6 \mathrm{meV}$ for the dissociation of CTSs. We also observed a long-time rise in PPPC response which we attribute to the charge trapping with a trap activation energy of $14 \pm 7 \mathrm{meV}$. Together with the reasonably high PL quenching yield, our results suggest that the main limiting factor in PffBT4T-2OD:EH-IDTBR performance is not the exciton dissociation but the ability of charges to escape from the D/A interface.

\section{EXPERIMENTAL SECTION}

\section{A. Materials}

PffBT4T-2OD and $\mathrm{PC}_{71} \mathrm{BM}$ were purchased from Ossila and 1-Material, respectively. EH-IDTBR was prepared according to the previously reported procedure. ${ }^{10}$

\section{B. Film preparation}

PffBT4T-2OD and $\mathrm{PC}_{71} \mathrm{BM}$ (D:A weight ratio 1:1.4) blend solution (polymer D concentration: $10 \mathrm{mg} \mathrm{ml}^{-1}$ ) was prepared in chlorobenzene $(\mathrm{CB})$ and dichlorobenzene $(\mathrm{DCB})$ (1:1 volume ratio) with 3 vol\% 1,8-diiodooctane. A PffBT4T-2OD and EH-IDTBR (D:A weight ratio 1:1.2) blend solution (polymer D concentration: $10 \mathrm{mg} \mathrm{ml}^{-1}$ ) was prepared in DCB. Active layer solutions were stirred on a hot plate at $110^{\circ} \mathrm{C}$ for $1 \mathrm{~h}$ and then at $60^{\circ} \mathrm{C}$ overnight. Prior to spin coating on to the preheated glass substrates $\left(80{ }^{\circ} \mathrm{C}\right)$ at $1500 \mathrm{rpm}$ in a nitrogen glovebox, the solutions were reheated to $110^{\circ} \mathrm{C}$.

\section{Device fabrication}

Devices were fabricated in an inverted architecture (ITO/ZnO/ PffBT4T-2OD:acceptor $/ \mathrm{MoO}_{3} / \mathrm{Ag}$ ). ITO substrates were cleaned with soap, water, acetone, and isopropanol. They were then treated with oxygen plasma prior to spin coating of zinc acetate dihydrate precursor solution ( $60.4 \mu \mathrm{l}$ 1-ethanolamine in $2 \mathrm{ml}$ 2-methoxyethanol). $\mathrm{ZnO}$ layers were annealed at $150^{\circ} \mathrm{C}$ for $10 \mathrm{~min}$. Blend solutions were prepared in the same way as previously described for film preparation. They were spun onto the ITO substrates at $1500 \mathrm{rpm}$ in a nitrogen glovebox. $\mathrm{MoO}_{3}(10 \mathrm{~nm})$ and $\mathrm{Ag}(100 \mathrm{~nm})$ layers were deposited by vacuum evaporation through a mask with an active area of $0.045 \mathrm{~cm}^{2}$ in each device. Current-voltage characteristics were measured with a Keithley 2400 source meter under AM 1.5 illumination from a xenon lamp (Oriel Instruments).

\section{UV-Vis absorbance spectroscopy}

A PerkinElmer Lambda 25 spectrometer was used to carry out UV-Vis absorbance for thin film samples.

\section{E. Steady state photoluminescence spectroscopy}

Steady-state PL spectra were measured on a Fluorolog-3 spectrofluorometer (FL 3-22, Horiba Jobin Yvon). All the samples were excited at $600 \mathrm{~nm}$ with a slit width of $5 \mathrm{~nm}$. The emitted photons were collected in the front-face geometry with a slit width of $5 \mathrm{~nm}$.

\section{F. Time correlated single photon counting}

The DeltaFlex TCSPC system (Horiba Scientific) was used to measure the PL kinetics of thin film samples. The samples were excited by a nanoLED at $635 \mathrm{~nm}$. Photons were detected with a picosecond photon detector (PPD) detector up to $950 \mathrm{~nm}$. The instrument temporal resolution is $100 \mathrm{fs}$.

\section{G. Transient absorption spectroscopy}

A broadband pump-probe fs TA spectrometer Helios (Spectra Physics, Newport Corp.) was used to measure the TA spectra and kinetics for thin film samples. Ultrafast laser pulses $(800 \mathrm{~nm}$, 100 fs pulse duration) were generated by using a $1 \mathrm{kHz}$ Ti:sapphire regenerative amplifier (Solstice, Spectra Physics). One portion of the $800-\mathrm{nm}$ pulses was directed to an optical parametric amplifier (TOPAS) to generate the visible pump pulses at $700 \mathrm{~nm}$. The rest of the $800 \mathrm{~nm}$ pulses routes onto a mechanical delay stage (6 ns time window) and is directed through a sapphire crystal to generate a white light probe ranging from 800 to $1600 \mathrm{~nm}$ in the near-infrared region. The pump and probe beams were focused onto the same spot on the samples. During the measurements, all the samples were kept in a quartz cuvette under continuous $\mathrm{N}_{2}$ flow.

\section{H. Pump-push-photocurrent spectroscopy}

fs-pulses (800 nm, $35 \mathrm{fs}$ ) were generated by using a $4 \mathrm{kHz}$ Ti:sapphire regenerative amplifier (Astrella, Coherent). These pulses are routed onto two optical parametric amplifiers (TOPAS Prime, Coherent). The $1200 \mathrm{~nm}$ output from one TOPAS passed through 
a frequency-doubling barium borate $(\mathrm{BBO})$ crystal to generate the pump at $600 \mathrm{~nm}$. The pump pulse was then directed onto a mechanical delay stage to vary the time delay between the pump and the push beams. The $2000 \mathrm{~nm}$ output from the other TOPAS serves as the push. The push was mechanically modulated at $1.1 \mathrm{kHz}$. Both the pump and push pulses were aligned onto a single spot on the device pixel. During the measurements, the devices were connected to a lock-in amplifier (SR830, Stanford Research Systems) and were measured under short-circuit conditions. The reference current $J$ is measured at the pump frequency of $4 \mathrm{kHz}$, and the push induced current $\Delta J$ was measured at the push frequency of $1.1 \mathrm{kHz}$.

\section{Global analysis}

Global analysis was carried out using a previously written programme based on a genetic algorithm. ${ }^{44}$ We first extract the spectra of singlet excitons of D and A from the TA measurement of the neat materials. As a time-efficient approach, we use those as initial guesses and add on randomness into each species. Throughout the fitting, the algorithm examines the fitness of each and reaches the most fitted result.

\section{SUPPLEMENTARY MATERIAL}

See supplementary material for time-resolved PL of neat materials and blend materials, time-resolved emission spectra of neat materials and the blend materials, normalised TA spectra for neat materials and the blend materials, and TEM of blend materials.

\section{ACKNOWLEDGMENTS}

The authors would like to thank Tom Hopper, Dr. Robert Godin, and Dr. Wenxing Yang for discussions. The authors would also like to thank Tom Hopper for proofreading the manuscript and Ahmad Alraddadi for TEM. The authors acknowledge the Optoelectronics Group in the University of Cambridge for sharing the software for global analysis. We thank the Welsh government funded Sêr Solar project and the UKRI Global Challenge Research Fund project SUNRISE (EP/P032591/1) for funding. A.A.B. is a Royal Society University Research Fellow. J.Z. thanks the China Scholarship Council for a Ph.D. scholarship (No. 201503170255).

The authors declare no competing financial interest.

\section{REFERENCES}

${ }^{1}$ G. Yu, J. Gao, J. C. Hummelen, F. Wudl, and A. J. Heeger, Science 270, 1789-1791 (1995).

${ }^{2}$ A. Wadsworth, M. Moser, A. Marks, M. S. Little, N. Gasparini, C. J. Brabec, D. Baran, and I. McCulloch, Chem. Soc. Rev. (published online, 2019).

${ }^{3}$ Y. Lin, J. Wang, Z.-G. Zhang, H. Bai, Y. Li, D. Zhu, and X. Zhan, Adv. Mater. 27, 1170-1174 (2015)

${ }^{4}$ C. Yan, S. Barlow, Z. Wang, H. Yan, A. K. Y. Jen, S. R. Marder, and X. Zhan, Nat. Rev. Mater. 3, 18003 (2018).

${ }^{5}$ J. Hou, O. Inganäs, R. H. Friend, and F. Gao, Nat. Mater. 17, 119-128 (2018).

${ }^{6}$ W. Zhao, D. Qian, S. Zhang, S. Li, O. Inganäs, F. Gao, and J. Hou, Adv. Mater. 28, 4734-4739 (2016).

${ }^{7}$ D. Sun, D. Meng, Y. Cai, B. Fan, Y. Li, W. Jiang, L. Huo, Y. Sun, and Z. Wang, J. Am. Chem. Soc. 137, 11156-11162 (2015).

${ }^{8}$ D. Baran, N. Gasparini, A. Wadsworth, C. H. Tan, N. Wehbe, X. Song, Z. Hamid, W. Zhang, M. Neophytou, T. Kirchartz, C. J. Brabec, J. R. Durrant, and I. McCulloch, Nat. Commun. 9, 2059 (2018).
${ }^{9}$ H. Cha, J. Wu, A. Wadsworth, J. Nagitta, S. Limbu, S. Pont, Z. Li, J. Searle, M. F. Wyatt, D. Baran, J.-S. Kim, I. McCulloch, and J. R. Durrant, Adv. Mater. 29, 1701156 (2017).

${ }^{10}$ S. Holliday, R. S. Ashraf, A. Wadsworth, D. Baran, S. A. Yousaf, C. B. Nielsen, C.-H. Tan, S. D. Dimitrov, Z. Shang, N. Gasparini, M. Alamoudi, F. Laquai, C. J. Brabec, A. Salleo, J. R. Durrant, and I. McCulloch, Nat. Commun. 7, 11585 (2016).

${ }^{11}$ L. Meng, Y. Zhang, X. Wan, C. Li, X. Zhang, Y. Wang, X. Ke, Z. Xiao, L. Ding, R. Xia, H.-L. Yip, Y. Cao, and Y. Chen, Science 361, 1094-1098 (2018).

${ }^{12}$ Z. Fei, F. D. Eisner, X. Jiao, M. Azzouzi, J. A. Röhr, Y. Han, M. Shahid, A. S. R. Chesman, C. D. Easton, C. R. McNeill, T. D. Anthopoulos, J. Nelson, and M. Heeney, Adv. Mater. 30, 1800728 (2018).

${ }^{13}$ F. Zhao, S. Dai, Y. Wu, Q. Zhang, J. Wang, L. Jiang, Q. Ling, Z. Wei, W. Ma, W. You, C. Wang, and X. Zhan, Adv. Mater. 29, 1700144 (2017).

${ }^{14}$ N. Gasparini, M. Salvador, S. Strohm, T. Heumueller, I. Levchuk, A. Wadsworth, J. H. Bannock, J. C. de Mello, H. J. Egelhaaf, D. Baran, I. McCulloch, and C. J. Brabec, Adv. Energy Mater. 7, 1700770 (2017).

${ }^{15}$ S. Zhang, Y. Qin, J. Zhu, and J. Hou, Adv. Mater. 30, e1800868 (2018).

${ }^{16}$ F. Gao, W. Tress, J. Wang, and O. Inganäs, Phys. Rev. Lett. 114, 128701 (2015).

${ }^{17}$ Y. Puttisong, Y. Xia, X. Chen, F. Gao, I. A. Buyanova, O. Inganäs, and W. M. Chen, J. Phys. Chem. C 122, 12640-12646 (2018).

${ }^{18}$ H. Bässler and A. Kohler, Phys. Chem. Chem. Phys. 17, 28451-28462 (2015).

${ }^{19}$ J. Kurpiers, T. Ferron, S. Roland, M. Jakoby, T. Thiede, F. Jaiser, S. Albrecht, S. Janietz, B. A. Collins, I. A. Howard, and D. Neher, Nat. Commun. 9, 2038 (2018).

${ }^{20}$ J. Benduhn, K. Tvingstedt, F. Piersimoni, S. Ullbrich, Y. Fan, M. Tropiano, K. A. McGarry, O. Zeika, M. K. Riede, C. J. Douglas, S. Barlow, S. R. Marder, D. Neher, D. Spoltore, and K. Vandewal, Nat. Energy 2, 17053 (2017).

${ }^{21}$ D. Qian, Z. Zheng, H. Yao, W. Tress, T. R. Hopper, S. Chen, S. Li, J. Liu, S. Chen, J. Zhang, X. K. Liu, B. Gao, L. Ouyang, Y. Jin, G. Pozina, I. A. Buyanova, W. M. Chen, O. Inganäs, V. Coropceanu, J. L. Bredas, H. Yan, J. Hou, F. Zhang, A. A. Bakulin, and F. Gao, Nat. Mater. 17, 703-709 (2018).

${ }^{22}$ T. M. Burke, S. Sweetnam, K. Vandewal, and M. D. McGehee, Adv. Energy Mater. 5, 1500123 (2015).

${ }^{23} \mathrm{~K}$. Vandewal, K. Tvingstedt, A. Gadisa, O. Inganäs, and J. V Manca, Nat. Mater. 8, 904-909 (2009).

${ }^{24}$ S. Chen, Y. Wang, L. Zhang, J. Zhao, Y. Chen, D. Zhu, H. Yao, G. Zhang, W. Ma, R. H. Friend, P. C. Y. Chow, F. Gao, and H. Yan, Adv. Mater. 30, 1804215 (2018).

${ }^{25}$ S. M. Menke, N. A. Ran, G. C. Bazan, and R. H. Friend, Joule 2, 25 (2017).

${ }^{26}$ Y. Liu, J. Zhao, Z. Li, C. Mu, W. Ma, H. Hu, K. Jiang, H. Lin, H. Ade, and H. Yan, Nat. Commun. 5, 5293 (2014).

${ }^{27}$ H. Cha, C.-H. Tan, J. Wu, Y. Dong, W. Zhang, H. Chen, S. Rajaram, K. S. Narayan, I. McCulloch, and J. R. Durrant, Adv. Energy Mater. 8, 1801537 (2018).

${ }^{28}$ L. Krückemeier, P. Kaienburg, J. Flohre, K. Bittkau, I. Zonno, B. Krogmeier, and T. Kirchartz, Commun. Phys. 1, 27 (2018).

${ }^{29}$ Y. Lin, F. Zhao, S. K. K. Prasad, J.-D. Chen, W. Cai, Q. Zhang, K. Chen, Y. Wu, W. Ma, F. Gao, J.-X. Tang, C. Wang, W. You, J. M. Hodgkiss, and X. Zhan, Adv. Mater. 30, 1706363 (2018).

${ }^{30}$ M. A. Alamoudi, J. I. Khan, Y. Firdaus, K. Wang, D. Andrienko, P. M. Beaujuge, and F. Laquai, ACS Energy Lett 3, 802-811 (2018).

${ }^{31}$ A. Weu, T. R. Hopper, V. Lami, J. A. Kreß, A. A. Bakulin, and Y. Vaynzof, Chem. Mater. 30, 2660-2667 (2018).

${ }^{32}$ I. A. Howard, R. Mauer, M. Meister, and F. Lacquai, J. Am. Chem. Soc. 132, 14866-14876 (2010).

${ }^{33}$ A. J. Ward, A. Ruseckas, M. M. Kareem, B. Ebenhoch, L. A. Serrano, M. Al-Eid, B. Fitzpatrick, V. M. Rotello, G. Cooke, and I. D. W. Samuel, Adv. Mater. 27, 2496-2500 (2015).

${ }^{34}$ J. L. Brédas, E. H. Sargent, and G. D. Scholes, Nat. Mater. 16, 35-44 (2016).

${ }^{35}$ F. Etzold, I. A. Howard, R. Mauer, M. Meister, T. D. Kim, K. S. Lee, N. S. Baek, and F. Laquai, J. Am. Chem. Soc. 133, 9469-9479 (2011).

${ }^{36}$ T. M. Clarke and J. R. Durrant, Chem. Rev. 110, 6736-6767 (2010).

${ }^{37}$ S. M. Menke, A. Cheminal, P. Conaghan, N. A. Ran, N. C. Greehnam, G. C. Bazan, T. Q. Nguyen, A. Rao, and R. H. Friend, Nat. Commun. 9, 277 (2018). 
${ }^{38}$ T. M. Clarke, A. M. Ballantyne, S. Tierney, M. Heeney, W. Duffy, I. McCulloch, J. Nelson, and J. R. Durrant, J. Phys. Chem. C 114, 8068-8075 (2010).

${ }^{39}$ A. A. Bakulin, A. Rao, V. G. Pavelyev, P. H. M. van Loosdrecht, M. S. Pshenichnikov, D. Niedzialek, J. Cornil, D. Beljonne, and R. H. Friend, Science 335, 1340-1344 (2012)

${ }^{40}$ Y. Liu, L. Zuo, X. Shi, A. K.-Y. Jen, and D. S. Ginger, ACS Energy Lett. 3, 23962403 (2018).

${ }^{41}$ J. Zhang, Q. Gu, T. T. Do, K. Rundel, P. Sonar, R. H. Friend, C. R. McNeill, and A. A. Bakulin, J. Phys. Chem. A 122, 1253-1260 (2018).
${ }^{42}$ J. Zhang, A. C. Jakowetz, G. Li, D. Di, S. M. Menke, A. Rao, R. H. Friend, and A. A. Bakulin, J. Mater. Chem. A 5, 11949-11959 (2017).

${ }^{43}$ A. A. Bakulin, S. Neutzner, H. J. Bakker, L. Ottaviani, D. Barakel, and Z. Chen, ACS Nano 7, 8771-8779 (2013).

${ }^{44}$ S. Gélinas, O. Paré-Labrosse, C. N. Brosseau, S. Albert-Seifried, C. R. McNeill, K. R. Kirov, I. A. Howard, R. Leonelli, R. H. Friend, and C. Silva, J. Phys. Chem. C 115, 7114-7119 (2011).

${ }^{45}$ A. C. Jakowetz, M. L. Böhm, J. Zhang, A. Sadhanala, S. Huettner, A. A. Bakulin, A. Rao, and R. H. Friend, J. Am. Chem. Soc. 138, 11672-11679 (2016). 